\title{
Fabrication and Simultaneous Electrical Measurement of Graphene Nanoribbon Devices Inside a S/TEM.
}

Julio A. Rodríguez-Manzo ${ }^{1}$, Zhengqing John Qi ${ }^{1}$, Matthew Puster ${ }^{1,2}$, Adrian Balan ${ }^{1}$, A. T. Charlie Johnson $^{1}$ and Marija Drndić ${ }^{1}$.

1. Department of Physics and Astronomy, University of Pennsylvania, Philadelphia, USA.

2. Department of Materials Science and Engineering, University of Pennsylvania, Philadelphia, USA.

The high-energy fine electron probe attainable in a transmission electron microscope (operated in TEM or STEM mode) could be thought of as an exquisite subtractive fabrication tool in terms of size $(<1 \mathrm{~nm})$ and spatial resolution $(<1 \mathrm{~nm})$. For devices that require drilling, cutting, stamping or molding at this level, and whose functionality truly stems from their nanoscale dimensions, the in-situ/operando S/TEM approach offers a top-notch platform where the physical properties (e.g., conductivity) of a material can be correlated with its structure and chemistry-with atomic resolution with the best conditions. The range of electron energies $(\sim 80-300 \mathrm{KeV})$ and the probe current density $\left(\sim 10^{9} \mathrm{~A} \mathrm{~m}^{-2}\right)$ attainable in a standard S/TEM equipped with a field emission gun are especially well suited to modify 2D materials. In this context, we discuss two examples in which graphene nanoribbon (GNR)-based devices were fabricated and modified with the electron probe while simultaneously electrically biased for electrical characterization.

Experiments were carried out in a FEI Titan (aberration-corrected objective lens) or a JEOL 2010F. GNR-based devices were prepared with standard lithographic techniques $[1,2]$ and mounted in sample holders containing electrical feedthroughs. For the latter we used either a home-made sample holder or commercially available sample holders from Protochips Inc. or Hummingbird Scientific.

In the first example, we describe how to fabricate GNR-nanopore devices, which are promising candidates for next-generation DNA sequencing [1]. Such devices normally comprise a nanopore with a diameter of 2-10 $\mathrm{nm}$ formed with the electron probe at the edge or in the center of a 100-nm-wide GNR on a 50-nm-thick silicon nitride membrane. We discuss the changes on GNR conductance when such devices are irradiated with $200 \mathrm{keV}$ electrons and the differences between irradiating with a homogenous (TEM mode) versus a scanned converged beam (STEM mode). By minimizing the average electron dose in STEM mode delivered to GNR to $\sim 10^{2} \mathrm{C} \mathrm{m}^{-2}$, we were able to minimize electron irradiation-induced damage and make nanopores in highly conducting GNR. The resulting devices, with unchanged resistances after nanopore formation, were tested outside the TEM column. They sustain micro ampere currents at low voltages $(\sim 50 \mathrm{mV})$ in buffered electrolyte solution and exhibit high sensitivity, with a large relative change of resistance upon changes of gate voltage, similar to pristine GNR without nanopores (Figure 1).

In the second example, we describe how to use the electron probe to sputter carbon atoms from predefined areas in electrically-connected free-standing graphene sheets to obtain GNR with widths $<10$ $\mathrm{nm}[2]$. We show cuts in graphene extending hundreds of nanometers with widths of a few nanometers, as well as different cut shapes (e.g., lines, sharp corners and circles). This approach allows us to correlate the lattice and edge structure of sub-10-nm wide GNR with their electrical properties (Figure 2). 
We believe these graphene-based examples illustrate the possibility to use the electron probe of a S/TEM to fabricate and modify 2D-materials-based devices with nanometer functional features and the advantages of performing in-situ S/TEM electrical measurements. Finally, we discuss some of the challenges (e.g., limiting leakage currents) involved in these types of experiments where chips fabricated with standard lithographic techniques are coupled to sample holders with electrical contacts and discuss different chip configurations suitable for the in-situ S/TEM electrical analysis of 2D materials.

\section{References:}

[1] M. Puster et al, ACS Nano 7 (2013), 11283.

[2] Z. J. Qi et al, Nano Letters 14 (2014), 4238.

[3] This work was supported by NIH grants R21HG004767 and R01HG006879, and NBIC through NSF NSEC DMR08-32802. JZQ and CJ acknowledge SRC contract \#2011-IN-2229, NSF AIR Program ENG-1312202. Part of this work was done at the CFN in BNL, supported by the U.S. DOE, Contract No. DE-AC02-98CH10886 (FEI-Titan ACTEM through proposal 31972).
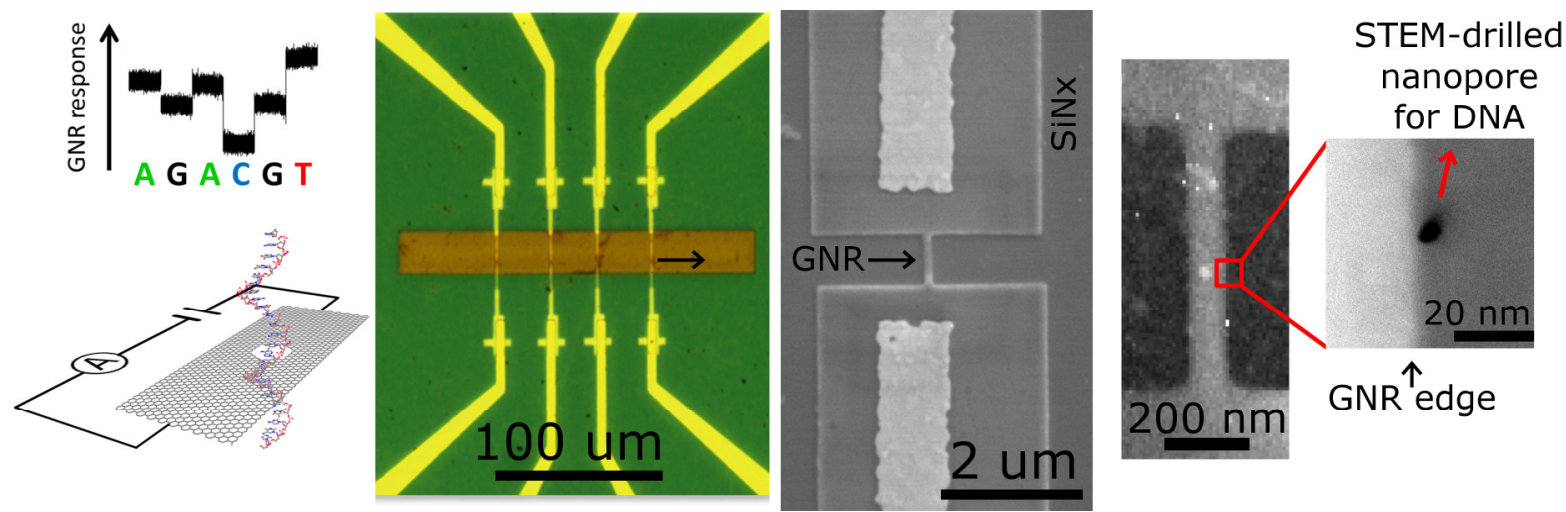

Figure 1. From left to right: scheme of GNR-nanopore device for DNA sequencing, optical image of TEM-compatible chip with 4 GNR-nanopore devices, SEM image of a GNR without a nanopore, and STEM image of a GNR-nanopore device.
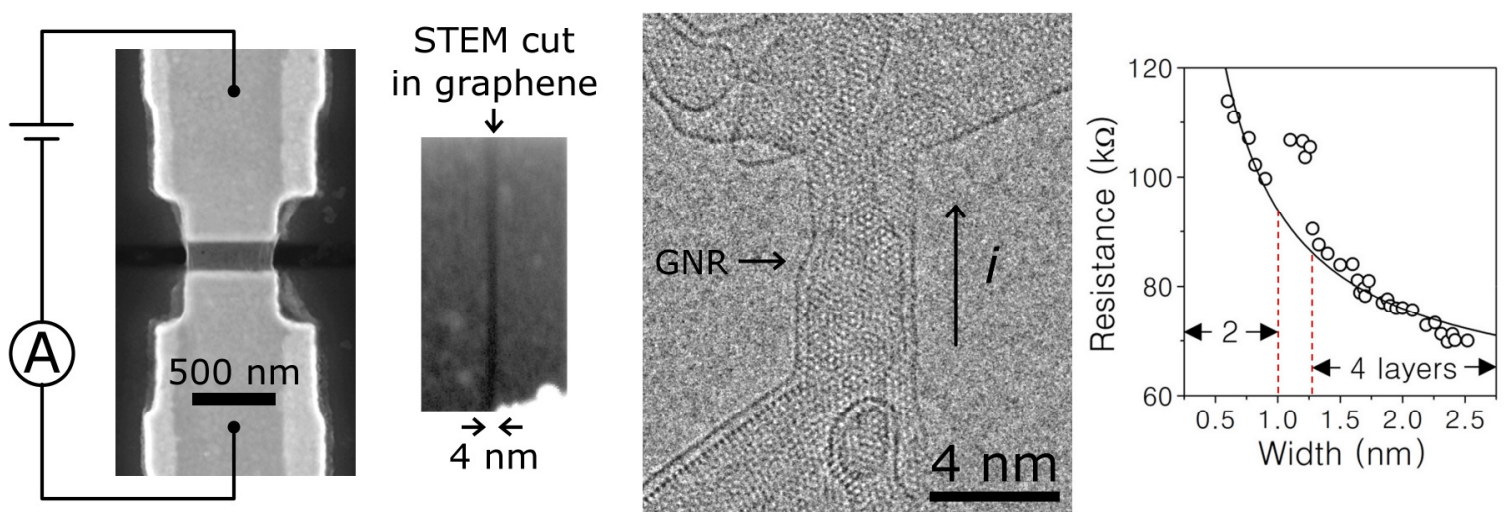

Figure 2. From left to right: SEM image of a GNR with electrical contacts, HAADF STEM image showing a straight cut made in STEM mode, HRTEM of a GNR, and GNR resistance as a function of width from data extracted from in-situ $\mathrm{S} / \mathrm{TEM}$ experiments. 